\title{
Preso regulates NMDA receptor-mediated excitotoxicity via modulating nitric oxide and calcium responses after traumatic brain injury
}

Peng Luo ${ }^{1,2}$, Xin $\mathrm{Li}^{3}$, Xiuquan $\mathrm{Wu}^{1}$, Shuhui Dai ${ }^{1}$, Yuefan Yang ${ }^{1}$, Haoxiang X ${ }^{1,2}$, Da Jing ${ }^{2}$, Wei Rao ${ }^{1}$, Hongyu Xu', Xiangyu Gao', Zhou Fei ${ }^{1}$ and Hongbing Lu $^{2}$

\begin{abstract}
Traumatic brain injury (TBI) has become a major health concern worldwide, and the poor outcome of TBI increases the need for therapeutic improvement. Secondary injuries following TBI, including excitotoxicity, lead to synaptic dysfunction and provide potential targets for intervention. Postsynaptic scaffold proteins, which are involved in the regulation of excitotoxicity after neuronal injury, play a crucial role in modulating synaptic function. Therefore, exploring the role of postsynaptic scaffold proteins in TBI might uncover new treatments. In this study, we demonstrated that downregulated expression of the postsynaptic scaffold protein Preso protects against neuronal injury after TBI in vitro and in vivo, and these effects are related to the inhibition of N-methyl-D-aspartate receptor (NMDAR) function. Further study showed that Preso facilitates signaling from NMDAR to nitric oxide (NO) and calcium $\left(\mathrm{Ca}^{2+}\right)$ responses. First, the complex constituting NMDAR, postsynaptic density-95 (PSD-95), and neuronal nitric oxide synthase (nNOS) was shown to be involved in the Preso regulation of the NO response. Uncoupling the linkage between Preso and PSD-95 attenuated the stability of this complex and suppressed the regulatory effect of Preso on the NO response. In addition, phosphorylation of NMDAR by cyclin-dependent kinase 5 (CDK5) was shown to be responsible for the Preso-mediated $\mathrm{Ca}^{2+}$ response, which was dependent on the interaction between Preso and CDK5. These results suggested that the association of Preso with NMDAR signaling can serve as a target for neuroprotection against TBI.
\end{abstract}

\section{Introduction}

Traumatic brain injury (TBI) is caused by external forces on the head, which cause closed or penetrating head injury and brain dysfunction. Due to its high mortality and morbidity, TBI has become a worldwide public health

\footnotetext{
Correspondence: Zhou Fei (zhoufei@fmmu.edu.cn) or

Hongbing Lu (luhb@fmmu.edu.cn)

'Department of Neurosurgery, Xijing Hospital, Fourth Military Medical University, Xi'an, China

${ }^{2}$ Department of Biomedical Engineering, Fourth Military Medical University, Xi'an, China

Full list of author information is available at the end of the article.

These authors contributed equally: Peng Luo, Xin Li, Xiuquan Wu, Shuhui Dai

Edited by D. Bano
}

problem and substantially burdens society ${ }^{1}$. Excitotoxicity is one of the most important mechanisms underlying the pathophysiological process of TBI. Activation of glutamate receptors by overproduction of glutamate at synapses contributes to the formation of excitotoxicity. Therefore, inactivation of glutamate receptors, including the N-methyl-D-aspartate receptor (NMDAR) and the metabotropic glutamate receptor (mGluR), by pharmacological antagonists has exhibited neuroprotective effects in animal experiments. However, these potential drugs did not show satisfactory curative effects on TBI patients in a series of clinical trials ${ }^{2}$.

\section{(c) The Author(s) 2019}

(c) (i) Open Access This article is licensed under a Creative Commons Attribution 4.0 International License, which permits use, sharing, adaptation, distribution and reproduction cc) in any medium or format, as long as you give appropriate credit to the original author(s) and the source, provide a link to the Creative Commons license, and indicate if changes were made. The images or other third party material in this article are included in the article's Creative Commons license, unless indicated otherwise in a credit line to the material. If material is not included in the article's Creative Commons license and your intended use is not permitted by statutory regulation or exceeds the permitted use, you will need to obtain permission directly from the copyright holder. To view a copy of this license, visit http://creativecommons.org/licenses/by/4.0/. 
Postsynaptic scaffold proteins are a special group of molecules belonging to postsynaptic density (PSD) proteins, which directly connect glutamate receptors with their postsynaptic signaling pathways ${ }^{3}$. Homer proteins are key postsynaptic scaffold proteins that regulate mGluR signaling via interactions with mGluR and its downstream mediators in the pathogenesis of TBI. The postsynaptic density-95 (PSD-95) protein is another important postsynaptic scaffold protein that binds NR2B, a subunit of NMDAR, together with neuronal nitric oxide synthase (nNOS). Recently, pharmacological inhibitors that uncouple PSD-95 from NMDAR signaling have been shown to reduce trauma-induced brain damage and neurological deficits ${ }^{4}$. These results indicated that postsynaptic scaffold proteins are potentially valuable for investigating the mechanism underlying the pathogenesis of TBI and potential targets for TBI interference.

PSD-95-interacting regulator of spine morphogenesis (Preso) is a novel scaffold protein that contains PSD-95/ DlgZOO1 (PDZ); 4.1, ezrin, radixin, and moesin (FERM); and WW domains ${ }^{5}$. Preso reportedly interacts with mGluR and Homer via its FERM domain and Homer ligand site to coordinate the function of mGluR by enhancing mGluR-Homer binding ${ }^{6}$. Furthermore, Preso binds with PSD-95, providing a link for NMDAR. Through these interactions with glutamate receptors, Preso regulates dendritic outgrowth and excitatory synaptic transmission and is further involved in the regulation of excitotoxicity ${ }^{7}$. Because of the crucial role of postsynaptic scaffold proteins in TBI, Preso provides a potential target for interfering with TBI. However, the role of Preso in TBI and how glutamate receptor signaling pathways are integrated for the Preso-dependent modulation of neuronal injury after TBI have not been fully elucidated. In this study, we showed that Preso, a novel postsynaptic scaffold protein, modulated NMDAR-related excitotoxicity after TBI. First, downregulation of Preso expression inhibited NMDAR-induced neurotoxicity and improved neuronal survival and recovery in models of TBI. Second, the effects of Preso on TBI were associated with its regulation of $\mathrm{NO}$ and $\mathrm{Ca}^{2+}$ responses induced by NMDAR. Third, Preso modulated NMDAR function after TBI by influencing the stability of the NR2B/PSD-95/ nNOS complex and NR2B phosphorylation.

\section{Results}

Dominant positive effects of Preso on traumatic neuronal injury

To investigate the effects of TBI on Preso, cortical neurons were used to establish a traumatic neuronal injury (TNI) model, an in vitro model of TBI (Fig. 1a). Preso expression was assessed by immunoblot at different time points. Preso expression remained stable after TNI (Fig. 1b). Then, lentiviral transduction was used to determine the role of Preso in neuronal survival and death after TNI (Fig. S1). Immunoblot analysis showed that a lentiviral-expressed shRNA targeting Preso (LV-shPreso) significantly decreased the expression of Preso (Fig. 1c), and a Preso-targeted lentivirus (LV-Preso) significantly increased the expression of Preso (Fig. 1f). Propidium iodide (PI) staining showed that LV-shPreso transfection reduced cell death at $12 \mathrm{~h}$ after TNI, while LV-Preso transfection exerted the opposite effect on neuronal cell death (Fig. 1d, g). In addition, a lactate dehydrogenase (LDH) release assay was used to analyze the cytotoxicity of neurons at $12 \mathrm{~h}$ after TNI. As shown in Fig. 1e, downregulation of Preso expression obviously decreased the release of LDH from neurons after TNI, while increased LDH release from neurons was observed after upregulation of Preso expression (Fig. 1h).

\section{Preso modulated NMDAR-related excitotoxicity after TNI}

Numerous studies have shown that glutamate receptormediated excitotoxicity plays crucial roles in TBI. To determine the effect of Preso on NMDAR-related excitotoxicity after TBI, cortical neurons were pretreated with the NMDAR-specific antagonists DL-AP5 $(100 \mu \mathrm{M})$ and MK-801 $(100 \mu \mathrm{M})$. Consistent with the data mentioned above, upregulation of Preso expression elevated the cell death rate and release of LDH at $12 \mathrm{~h}$ after TNI. These effects were prevented by pretreatment with DL-AP5 and MK-801 (Fig. 2a, b). Furthermore, NMDA was used to induce excitotoxicity in mouse cortical neurons. Downregulation of Preso expression by LV-shPreso reduced neuronal injury after NMDAR activation (Fig. 2c, d). However, alteration of Preso did not affect the expression of NMDAR subunits, including NR1, NR2A, and NR2B (Fig. 2e-h). These results suggested that Preso modulated the function of NMDAR by means other than affecting NMDAR expression.

\section{Preso regulated $\mathrm{Ca}^{2+} / \mathrm{NO}$ responses mediated by NMDAR- related excitotoxicity}

One possible explanation for the modulation of NMDAR function by Preso might be related to calcium responses or subsequent NO generation. To determine the role of Preso in regulating $\mathrm{Ca}^{2+}$ mobilization and NO production after TNI, we used established probes for free $\mathrm{Ca}^{2+}$ (Fura-2a) and NO (DAF-FM). Downregulation of Preso expression inhibited $\mathrm{Ca}^{2+}$ overload and NO production, while upregulation of Preso expression elevated $\mathrm{Ca}^{2+}$ levels and NO responses (Fig. 3a-c). The effects of Preso upregulation on $\mathrm{Ca}^{2+}$ and $\mathrm{NO}$ responses were suppressed by pretreatment with the NMDAR antagonists DL-AP5 and MK-801 (Fig. 3d, e). To further clarify the role of $\mathrm{Ca}^{2+} / \mathrm{NO}$ responses in Preso-mediated neuronal injury, we used BAPTA-AM $(10 \mu \mathrm{M})$ and ARL 17447 $(20 \mu \mathrm{M})$ to eliminate intracellular $\mathrm{Ca}^{2+}$ and inhibit $\mathrm{NO}$ 


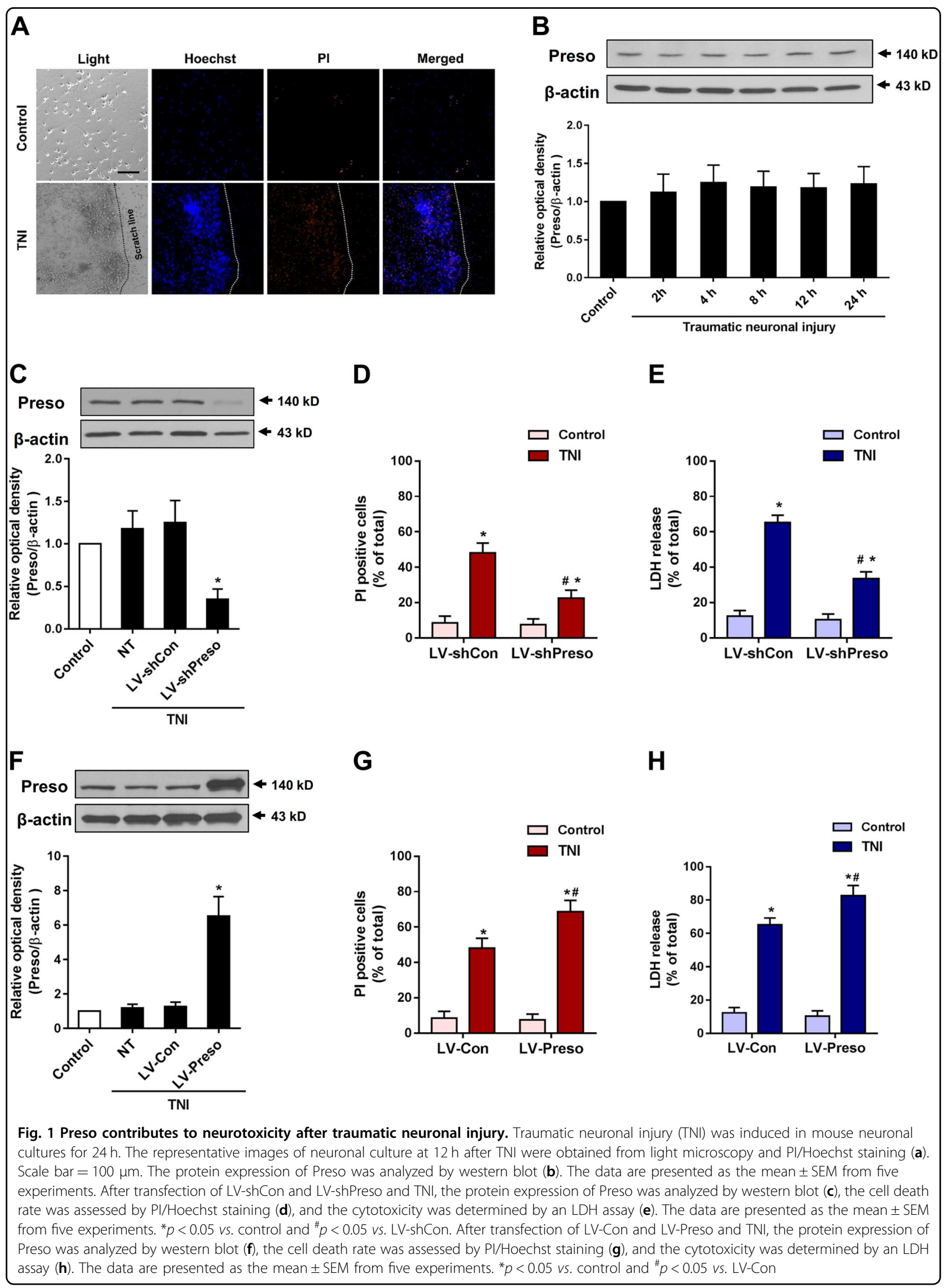



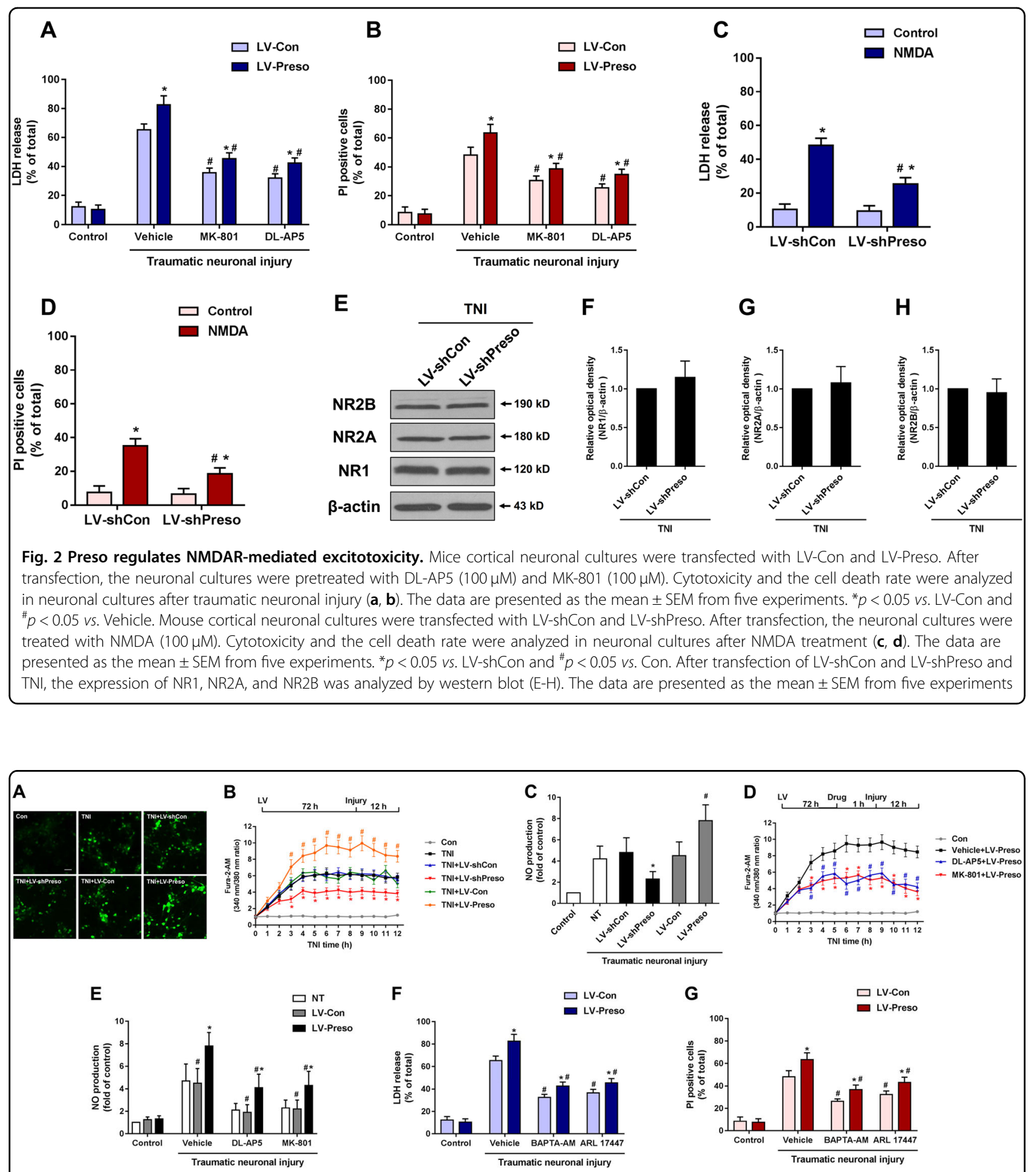

Fig. 3 Regulation of NMDAR-related $\mathrm{Ca}^{2+}$ /NO responses by Preso. Mouse cortical neuronal cultures were transfected with different lentiviruses. After transfection, the intracellular $\mathrm{Ca}^{2+}$ concentrations and NO production were analyzed after traumatic neuronal injury for $12 \mathrm{~h}(\mathbf{a}-\mathbf{c})$. The data are presented as the mean \pm SEM from five experiments. ${ }^{*} p<0.05$ vs. LV-shCon and ${ }^{\#} p<0.05$ vs. LV-Con. Scale bar $=50 \mu m$. Mouse cortical neuronal cultures were transfected with LV-Con and LV-Preso. After transfection, the neuronal cultures were pretreated with DL-AP5 (100 $\mu \mathrm{M})$ and MK-801 $(100 \mu \mathrm{M})$. The intracellular $\mathrm{Ca}^{2+}$ concentrations and $\mathrm{NO}$ production were analyzed at $12 \mathrm{~h}$ after TNI (D-E). The data are presented as the mean \pm SEM from five experiments. ${ }^{*} p<0.05 \mathrm{vs}$. LV-Con and ${ }^{\#} p<0.05 \mathrm{vs}$. Vehicle. After transfection, the neuronal cultures were pretreated with BAPTA-AM (10 $\left.\mu \mathrm{M}\right)$ and ARL $17447(20 \mu M)$. Cytotoxicity and the cell death rate were analyzed in neuronal cultures at $12 \mathrm{~h}$ after TNI (E-F). The data are presented as the mean \pm SEM from five experiments. ${ }^{*} p<0.05$ vs. LV-Con and ${ }^{\#} p<0.05$ vs. Vehicle 


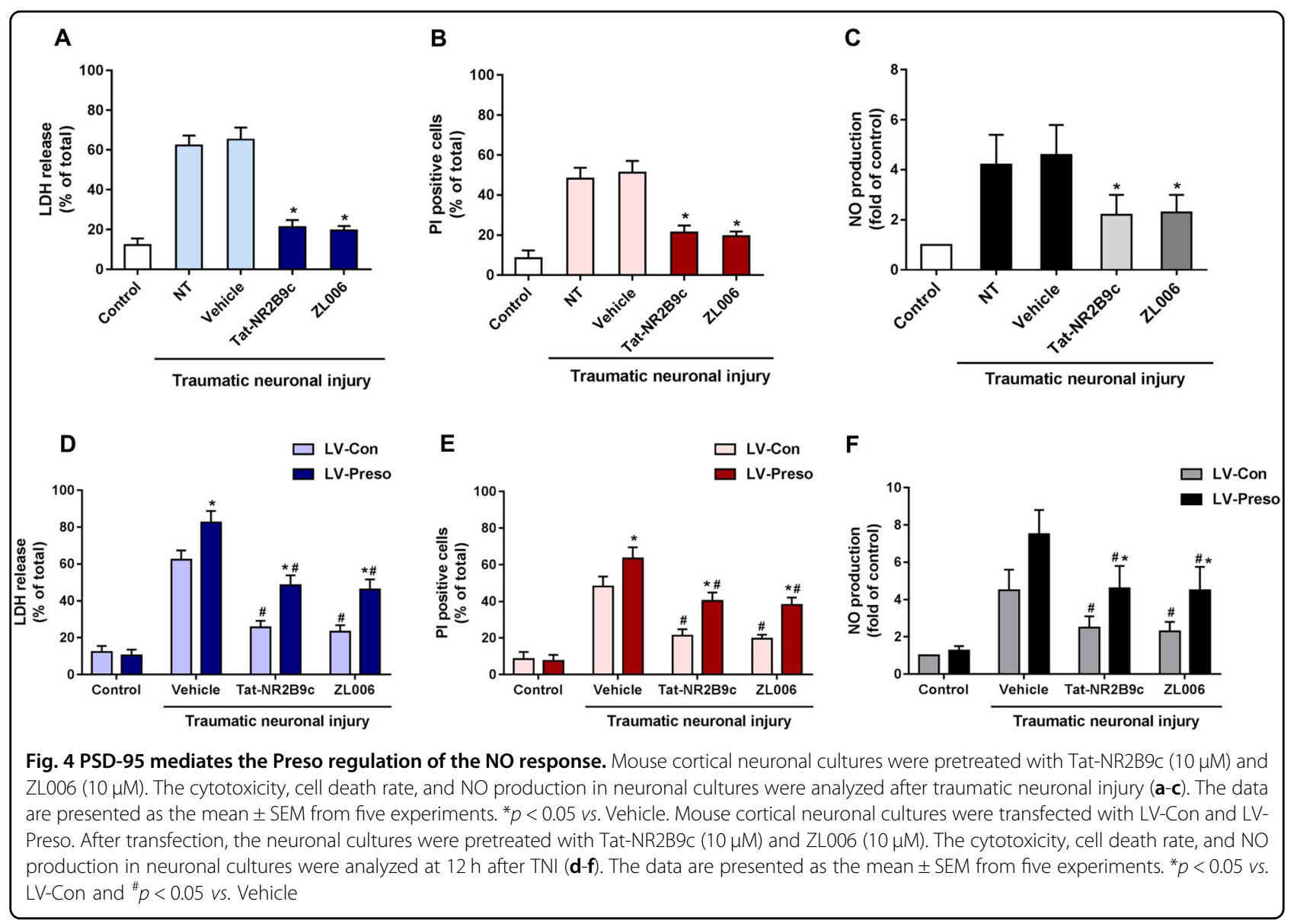

production. Pretreatment with BAPTA-AM and ARL 17447 reduced the elevation of LDH release and cell death induced by upregulation of Preso expression at $12 \mathrm{~h}$ after TNI (Fig. 3f, g). Therefore, these results indicate that Preso-mediated $\mathrm{Ca}^{2+} / \mathrm{NO}$ responses contribute to NMDAR-related excitotoxicity after TBI.

\section{Interaction between Preso and PSD-95 regulated the NMDAR-induced NO response}

PSD-95 is an important scaffold protein that facilitates signaling from NMDAR to $\mathrm{NO}$ by formation of the NR2B/PSD-95/nNOS complex. To investigate the role of PSD-95 in Preso-mediated neuronal injury and NO response, we used two antagonists specific for PSD-95, Tat-NR2B9c (to disrupt the interaction between NR2B and PSD-95) and ZL006 (to disrupt the interaction between PSD-95 and nNOS). As expected, Tat-NR2B9c $(10 \mu \mathrm{M})$ and ZL006 $(10 \mu \mathrm{M})$ reduced the neurotoxicity and inhibited the $\mathrm{NO}$ response at $12 \mathrm{~h}$ after TNI (Fig. 4a-c). These two drugs also suppressed the increased LDH release, cell death, and NO production induced by upregulated Preso expression following TNI (Fig. 4d-f).
To further clarify the mechanism by which Preso regulates the NR2B/PSD-95/nNOS complex, we assessed the role of Preso in modulating protein expression and the protein-protein interactions of the NR2B/PSD-95/nNOS complex. Similar to those of NR2B, the expression levels of PSD-95 and nNOS were not altered by upregulation of Preso expression (Fig. 5a-c). However, downregulation of Preso expression disrupted the interactions between PSD95/NR2B and PSD-95/nNOS after excitotoxicity. In contrast, upregulation of Preso expression enhanced the PSD95/NR2B and PSD-95/nNOS interactions (Fig. 5d-f). Because previous studies have shown that Preso has a PSD-95 binding site at its C-terminus, we constructed a lentiviral vector containing the Preso mutant variant lacking the PSD-95-binding C-terminus (LV-Preso $\Delta C$ ). After deletion of the C-terminal PDZ domain, Preso did not increase the PSD-95/NR2B and PSD-95/nNOS interactions (Fig. $5 \mathrm{~d}-\mathrm{f}$ ), and its effects on the NO response and neurotoxicity were also significantly reduced (Fig. $5 \mathrm{~g}-\mathrm{i}$ ). These results indicated that Preso regulated the NMDAR-induced $\mathrm{NO}$ response by influencing the NR2B/PSD-95/nNOS complex. 


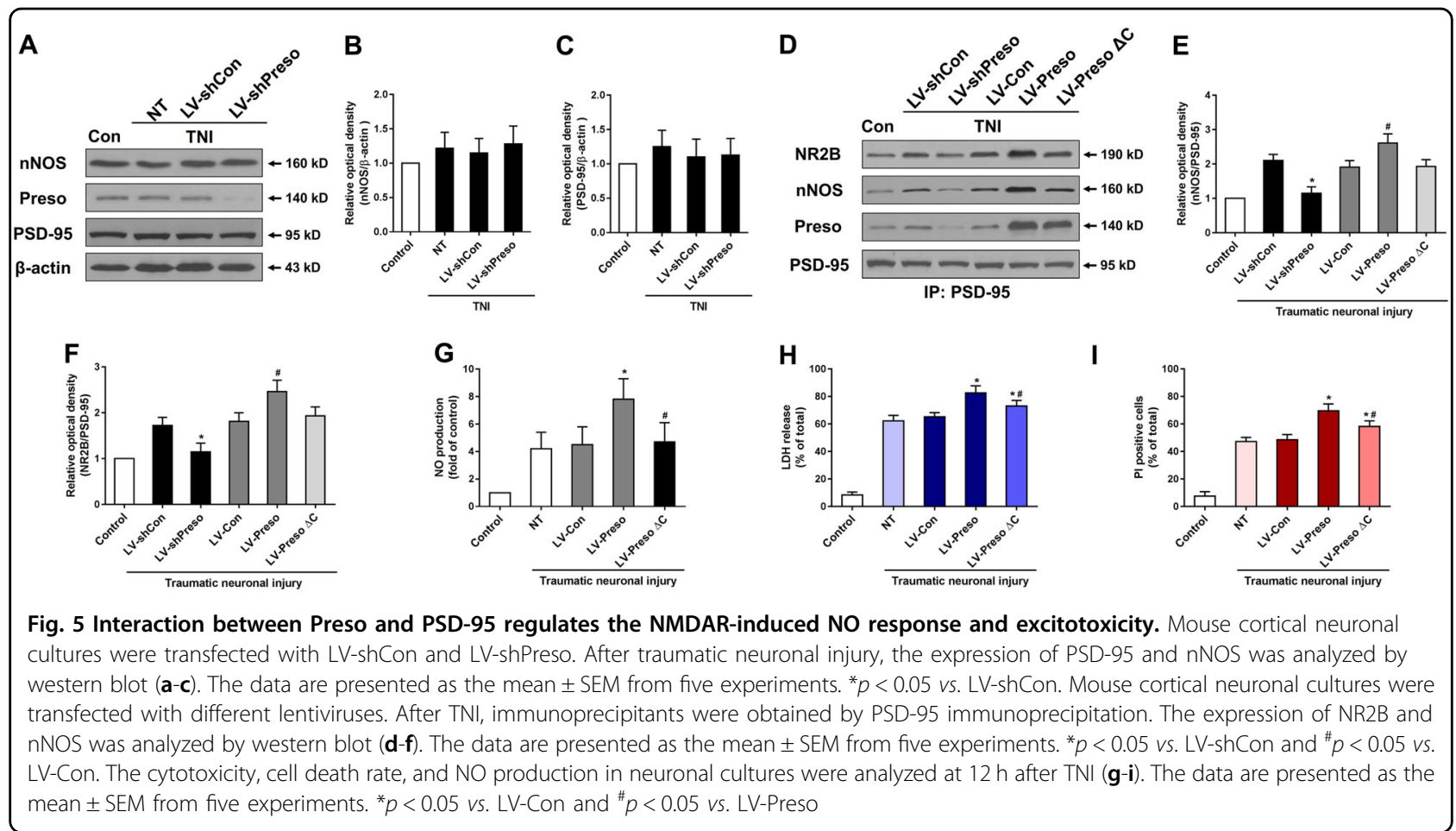

Involvement of CDK5 in the Preso regulation of the NMDAR-related $\mathrm{Ca}^{2+}$ response

CDK5 has been shown to regulate the function of NMDAR and the $\mathrm{Ca}^{2+}$ response after brain injury. To examine the relationship between Preso and CDK5 after traumatic neuronal injury, cortical neurons were transfected with LV-Preso, LV-shPreso, and their control viruses. Upregulation of Preso expression increased CDK5 activity, while downregulation of Preso expression decreased CDK5 activity (Fig. 6a). After treatment with purvalanol B $(50 \mu \mathrm{M})$, a CDK5 antagonist, the neuronal injury and $\mathrm{Ca}^{2+}$ overload induced by upregulated Preso expression were suppressed at $12 \mathrm{~h}$ after TNI (Fig. 6b).

Next, we determined the role of CDK5 in the NMDAR-related $\mathrm{Ca}^{2+}$ response after TNI. After the transfection of lentivirus expressing CDK5 (LV-CDK5), the $\mathrm{Ca}^{2+}$ response induced by TNI was increased (Fig. 6c). This effect was reduced by the administration of DL-AP5 (100 $\mu \mathrm{M})$, an NMDAR antagonist (Fig. 6d). Inhibition of CDK 5 activity by purvalanol $B$ reduced the phosphorylation of NR2B at S1284 at $12 \mathrm{~h}$ after TNI (Fig. 7a). Downregulation of Preso expression reduced the phosphorylation of NR2B, while upregulation of Preso expression enhanced the phosphorylation of NR2B (Fig. 7b). Because Preso contains a D-domain, which acts as a binding site for proline-directed kinase, we constructed a lentiviral vector containing the Preso mutant variant lacking the D-domain (LV-Preso $\Delta \mathrm{D}$ ). After deletion of the D-domain, the effects of Preso on the phosphorylation of NR2B and CDK5 activity were significantly attenuated (Fig. 7c, d), and its effects on the $\mathrm{Ca}^{2+}$ response were also suppressed (Fig. 7e). These results suggested that the NMDAR-related $\mathrm{Ca}^{2+}$ response was modulated by Preso through its interaction with CDK5.

\section{Downregulation of Preso expression improved recovery after TBI}

To further clarify whether Preso has similar effects in vivo, we used a controlled cortical impact (CCI) model to mimic TBI in vivo (Fig. 8a). We infused adenoassociated viruses containing shRNA Preso (AAVshPreso) into the unilateral cortices of mice (Fig. S2). Two weeks later, brain trauma was induced in the cortex ipsilateral to the AAV-shPreso or AAV-shCon injection site, and the brain water content was measured at $1,3,5$, and $7 \mathrm{~d}$ after TBI. The mouse neurological severity scores (NSSs) were assessed for seven consecutive days after injury. Downregulation of Preso expression in the lesion hemisphere significantly reduced brain edema and neurological deficits (Fig. 8b, c). Similar to data obtained from the in vitro studies, interactions of PSD-95/NR2B and PSD-95/nNOS were disrupted in lesions infected by AAV-shPreso (Fig. 8d-f). Disruption of PSD-95/NR2B and PSD-95/nNOS by Tat-NR2B9c and ZL006, respectively, improved recovery after TBI (Fig. 8g, h). NR2B phosphorylation and CDK5 activity was reduced by the administration of AAV-shPreso (Fig. 8i, j). Inhibition of 
A
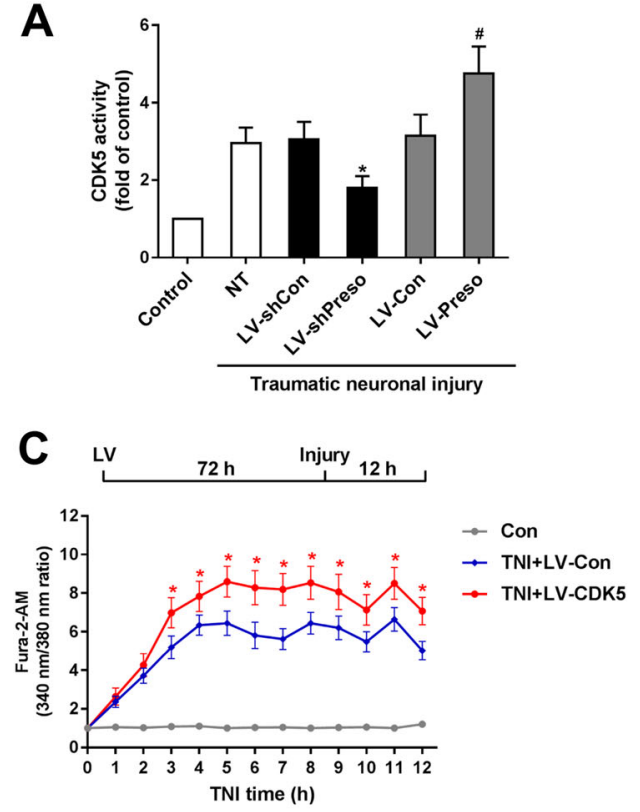
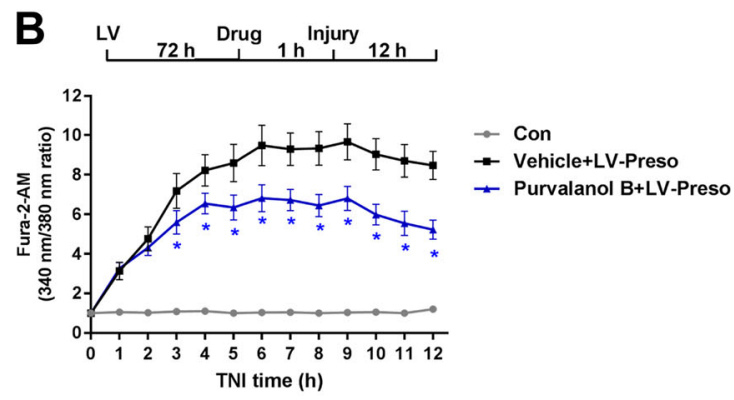

D
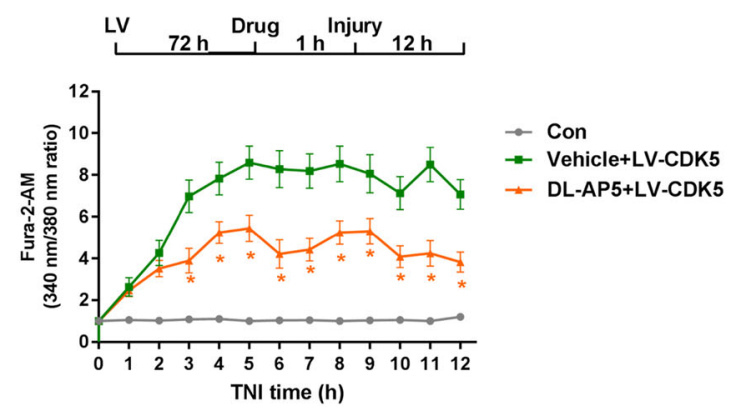

Fig. 6 Involvement of CDK5 in the Preso regulation of the NMDAR-related $\mathrm{Ca}^{2+}$ response. Mouse cortical neuronal cultures were transfected with different lentiviruses. CDK5 activity was analyzed after traumatic neuronal injury (a). The data are presented as the mean \pm SEM from five experiments. ${ }^{*} p<0.05$ vs. LV-shCon and ${ }^{*} p<0.05$ vs. LV-Con. After transfection with LV-Preso, the neuronal cultures were pretreated with purvalanol B $(50 \mu \mathrm{M})$. The intracellular $\mathrm{Ca}^{2+}$ concentrations were analyzed at $12 \mathrm{~h}$ after TNI (b). The data are presented as the mean \pm SEM from five experiments. ${ }^{*} p<0.05$ vs. Vehicle. Mouse cortical neuronal cultures were transfected with LV-Con and LV-CDK5. The intracellular Ca ${ }^{2+}$ concentrations were analyzed at $12 \mathrm{~h}$ after TNI (c). The data are presented as the mean \pm SEM from five experiments. ${ }^{*} p<0.05 \mathrm{vs}$. LV-Con. After transfection, the neuronal cultures were pretreated with DL-AP5 $(100 \mu \mathrm{M})$. The intracellular $\mathrm{Ca}^{2+}$ concentrations were analyzed at $12 \mathrm{~h}$ after TNI (d). The data are presented as the mean \pm SEM from five experiments. ${ }^{*} p<0.05$ vs. Vehicle

CDK5 activity by purvalanol $\mathrm{B}$ attenuated brain edema and neurological deficits (Fig. 8k, l). Overall, these results suggested that positive regulation of the NR2B/PSD-95/ nNOS complex and CDK5 activity by Preso plays an important role in brain damage after TBI.

\section{Discussion}

Postsynaptic scaffold proteins act as crucial coordinators of synaptic function due to their interaction with synaptic receptors and their intracellular mediators. As a postsynaptic scaffold protein with FERM, WW, Homer ligand, and PDZ domains, Preso directly links with G protein-coupled receptors (mGluR), other scaffold proteins (PSD-95 and Homer), PAK-interacting exchange factor-beta ( $\beta$ Pix), membrane phosphatidylinositol 4,5bisphosphate (PIP2), proline-directed kinases (CDK5 and ERK), and the cytoskeleton and has been reported to regulate dendritic spine morphogenesis and the inflammatory pain response $5,6,8,9$. Our present study showed that inhibition of Preso expression suppressed neuronal injury and prevented brain damage after TBI, demonstrating that downregulating Preso expression exerts an incremental neuroprotective effect on TBI. This effect of Preso on TBI might be associated with its role in regulating excitotoxicity. Accordingly, the ability of Preso to mediate excitotoxicity raises the question of whether a similar Preso function is involved in other mechanisms following TBI.

Glutamate receptors play an important role in regulating neuronal death and survival following TBI. Postsynaptic scaffolds constitute a molecular framework at the postsynaptic level that provides a platform for synaptic transmission related to glutamate receptors. Therefore, the interaction between glutamate receptors and postsynaptic scaffold proteins contributes to the modulation of neuronal injury after TBI. Disruption of the interaction between PSD-95 and NR2B, a NMDAR subunit, reduced brain damage and improved neurological dysfunction after $\mathrm{TBI}^{4,10}$. Furthermore, Homer 1a attenuated neuronal injury and brain damage after TBI by differentially regulating group I mGluRs ${ }^{11}$. Our previous study showed that Preso affects neuronal injury related to NMDAR activation following glutamate-induced excitotoxicity ${ }^{7}$. After investigating the relationship between Preso and glutamate receptors in TBI, we herein showed that Preso serves as a crucial mediator of NMDAR signaling, which is in agreement with our previous study on excitotoxic neuronal injury. 


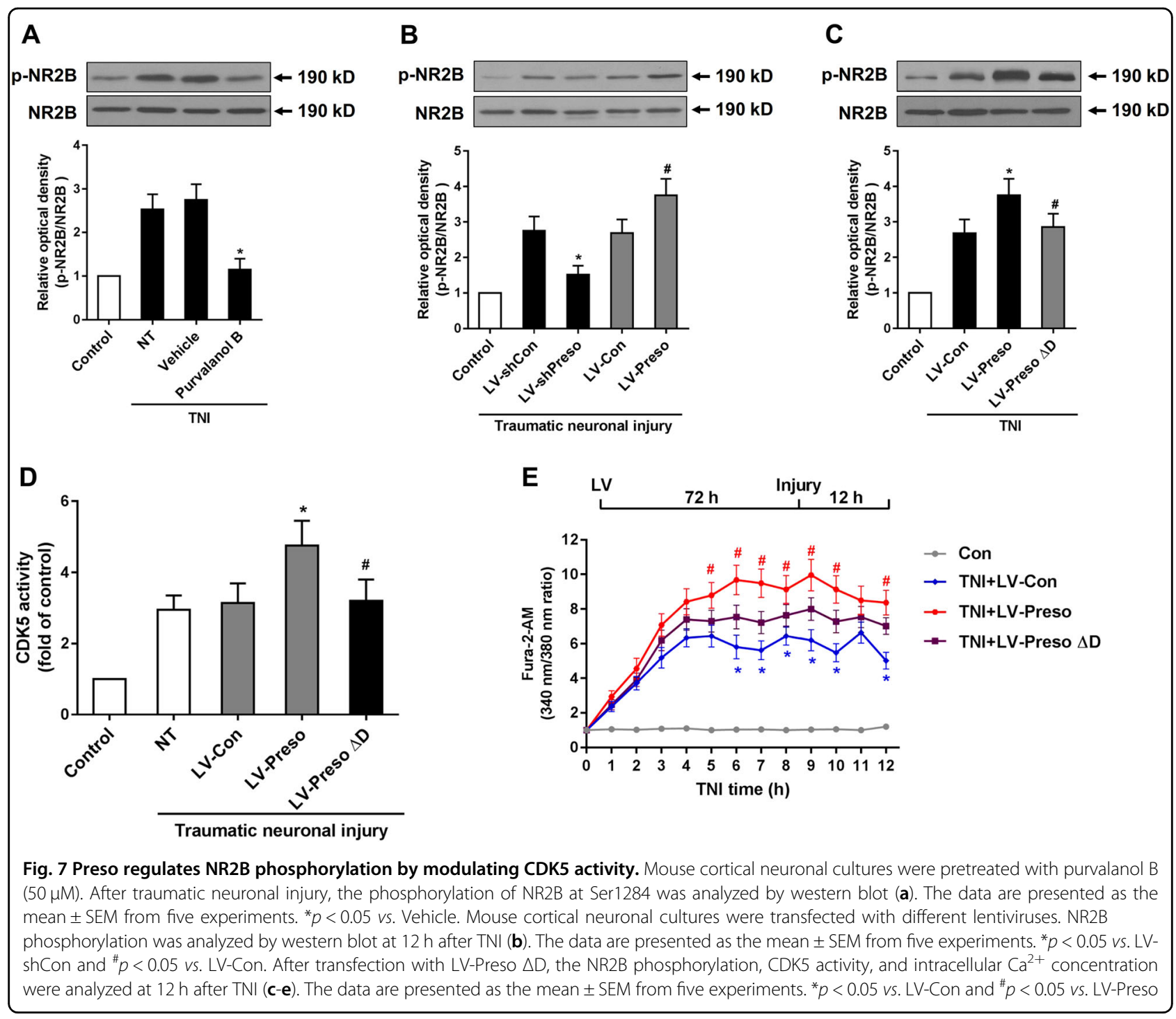

The overload of intracellular $\mathrm{Ca}^{2+}$ by excessive NMDAR activation is regarded as the key mechanism underlying secondary brain injury following $\mathrm{TBI}^{12}$. This regulation of $\mathrm{Ca}^{2+}$ redistribution is related to the ion channel function of NMDAR at the postsynaptic membrane. The NMDAR-induced overload of $\mathrm{Ca}^{2+}$ participates in the activation of intracellular signaling pathways responsible for neuronal injury and dysregulation of synaptic plasticity. Furthermore, activation of NMDAR induces the overproduction of $\mathrm{NO}$, resulting in nitrative stress and mitochondrial dysfunction after $\mathrm{TBI}^{13}$. This process is associated with modulation of $\mathrm{nNOS}$ by NMDAR activation ${ }^{14}$. In a previous study, we confirmed that Preso regulates NMDAR-related $\mathrm{Ca}^{2+}$ mobilization, suggesting that it exerts regulatory effects on the ion channel function of NMDAR ${ }^{7}$. In the present study, we further affirmed that Preso promotes not only $\mathrm{Ca}^{2+}$ overload but also $\mathrm{NO}$ overproduction by regulating
NMDAR after TBI. Therefore, it is important to further clarify the mechanism by which Preso modulates NMDAR-induced $\mathrm{Ca}^{2+} / \mathrm{NO}$ responses in TBI.

Postsynaptic signaling transduction from NMDAR to $\mathrm{NO}$ is dependent on formation of the NR2B/PSD-95/ nNOS complex ${ }^{15-17}$. Recently, uncoupling the formation of the NR2B/PSD-95/nNOS complex with pharmacological inhibitors has shown a promising neuroprotective effect ${ }^{18-20}$. In our previous study, regulation of Preso did not change the expression of the NMDAR subunits or influence their surface distribution, demonstrating that Preso may not be related to the expression and trafficking of NMDAR ${ }^{7}$. Like NR2B, Preso also did not affect the expression of PSD-95 and nNOS in the present study (Fig. S3). Overall, the regulatory effects of Preso on TBI via the NR2B/PSD-95/nNOS complex are not associated with changes in the components of this complex. Our subsequent study showed that downregulation of Preso 


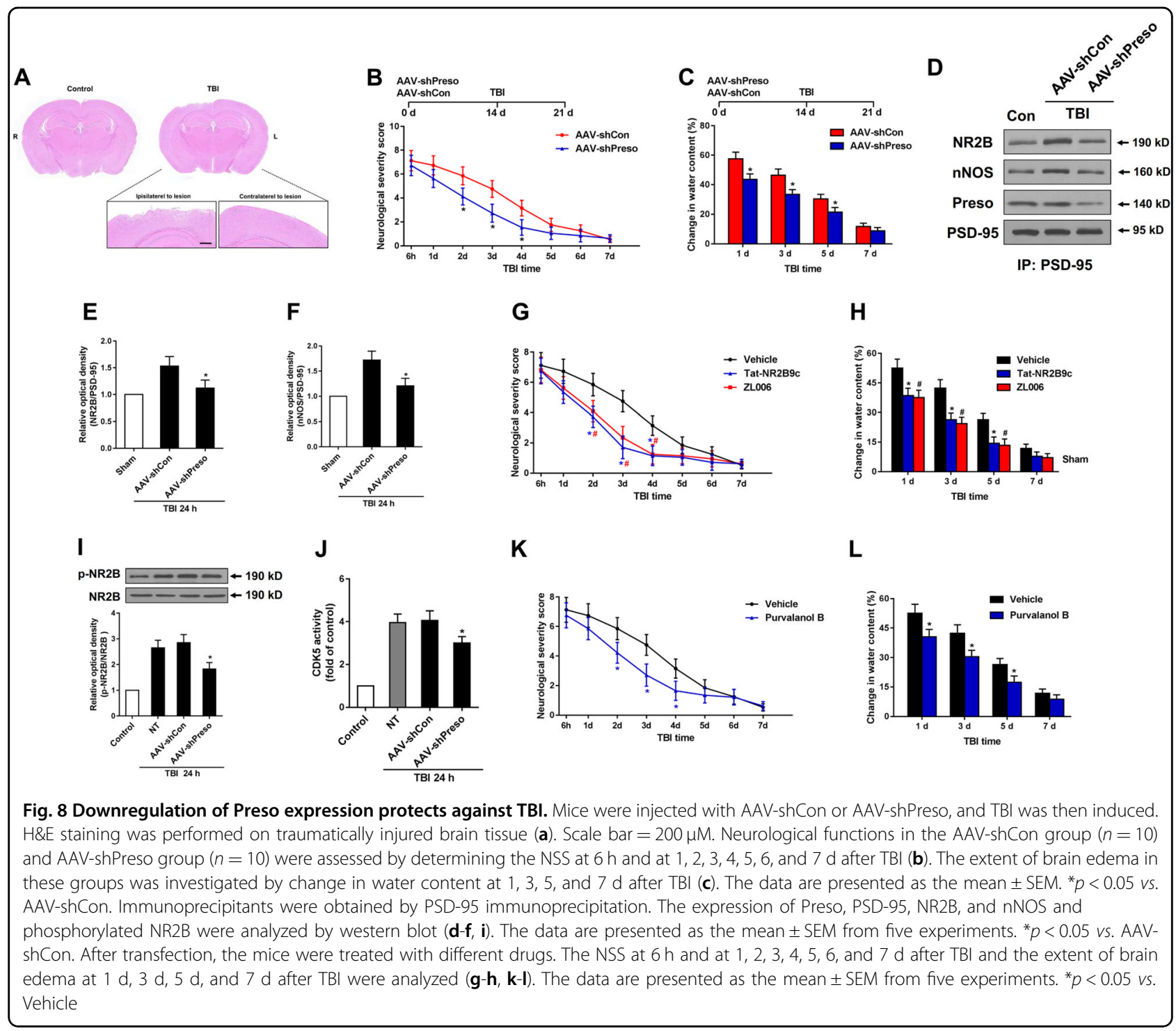

expression disrupts the NR2B/PSD-95/nNOS complex by reducing the interaction of PSD-95 with NR2B and the interaction of PSD-95 with nNOS. Structurally, the Cterminal PDZ binding domain of Preso provides a binding site for PSD-95 ${ }^{5}$. Compared with Preso without any mutations, the C-terminal mutant of Preso did not affect the formation of the NR2B/PSD-95/nNOS complex, suggesting that the binding of Preso to PSD-95 plays a crucial role in NR2B/PSD-95/nNOS complex regulation. This phenomenon of Preso promoting the formation of the NR2B/PSD-95/nNOS complex might be associated with its ability to enhance the stability of PSD-95 at the postsynaptic level.

CDK5 is a proline-directed serine/threonine kinase that plays an important role in synaptic plasticity and neurological diseases ${ }^{21}$. Aberrant CDK5 activity has been shown to be a principle cause of neuronal cell death during $\mathrm{TBI}^{22}$. Consistent with a previous study, we found that Preso positively regulated CDK5 activity, contributing to the neurotoxicity and overload of $\mathrm{Ca}^{2+}$ after traumatic injury. Recent studies have reported that NMDAR phosphorylation exerts various regulatory effects on receptor function, including synaptic trafficking and $\mathrm{Ca}^{2+}$ permeability $^{23,24}$. CDK5 has been implicated in the phosphorylation of certain serine sites on NMDARs. Phosphorylation of NR2A at serine 1232 by CDK5 leads to enhanced NMDAR function in neuronal cell death ${ }^{25}$. CDK5 modulates the surface expression of NR2B and memory formation through its phosphorylation of NR2B at serine $1116^{26}$. In the present study, we found that Preso modulated the phosphorylation of NR2B at serine 1284 by regulating CDK5. Although this NR2B phosphorylation 


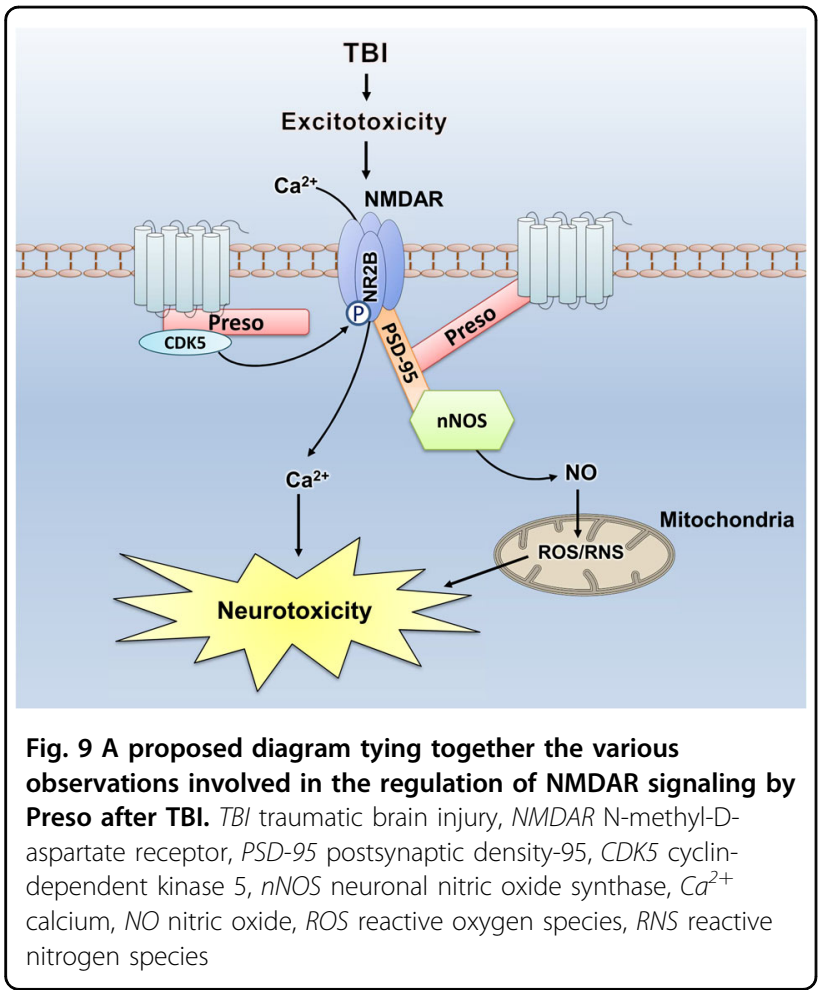

site was previously reported ${ }^{27,28}$, its role in TBI remained unclear. Thus, we demonstrated that the phosphorylation of NR2B at serine 1284 is tightly associated with the NMDAR-induced $\mathrm{Ca}^{2+}$ response after TBI. This regulatory pattern was shown to be dependent on the Preso D-domain, which is a binding site for proline-directed kinases ${ }^{6}$.

In the present study, we elucidated the mechanism by which Preso modulates NMDAR-related excitotoxicity after TBI (Fig. 9). However, the role of Preso in TBI and its underlying mechanisms may not be limited to the regulation of NMDAR signaling. In previous studies, mGluR signaling has been reported to be another important target of Preso in the regulation of synaptic function. Preso binds mGluR via its FERM domain and contains a Homer protein binding site ${ }^{6,9}$. Because our previous studies showed the crucial effects of mGluR and Homer on TBI, Preso is expected to regulate TBI via its interaction with mGluR-Homer signaling. Moreover, the characteristic structure of Preso provides various proteinprotein interaction patterns and targets for interfering with different intracellular signaling pathways. Compounds targeting these binding sites might accurately regulate Preso and its downstream mediators, thereby specifically interfering with synaptic function and neurological diseases. Therefore, further investigation is required to elucidate the mechanism of Preso in neurological diseases and elucidate novel targets for these diseases.

\section{Materials and methods}

\section{Animals}

C57BL/6 mice (10-12 weeks, 25-28 g), obtained from the Experimental Center of Fourth Military Medical University, were maintained at a constant temperature (approximately $27^{\circ} \mathrm{C}$ ) in an air-conditioned room for at least $7 \mathrm{~d}$ before the study and exposed to a 12-h light/dark cycle. All animal studies were performed in adherence with the National Institutes of Health Guidelines for the Care and Use of Laboratory Animals and approved by the Fourth Military Medical University Committee on Animal Care.

\section{Primary culture of cortical neurons}

Neuronal cortical cultures were prepared as previously described with some modifications ${ }^{29}$. Briefly, cerebral cortices were removed from embryos at 16-18 d. Tissues were dissociated by $0.25 \%$ trypsin for $15 \mathrm{~min}$ at $37^{\circ} \mathrm{C}$ and gentle trituration. Neurons were resuspended in neurobasal medium containing 2\% B27 supplement and $0.5 \mathrm{mM}$ L-glutamine (Thermo Fisher Scientific, Rockford, IL, USA) and plated at a density of $3 \times 10^{5}$ cells $/ \mathrm{cm}^{2}$. Before seeding, culture vessels consisting of 96-well plates, 1.5$\mathrm{cm}$ glass slides or $6-\mathrm{cm}$ dishes were coated with poly-Llysine $(50 \mu \mathrm{g} / \mathrm{mL})$ at room temperature overnight. The neurons were maintained at $37^{\circ} \mathrm{C}$ in a humidified $5 \% \mathrm{CO}_{2}$ incubator, and half of the culture medium was changed every other day. The cultured neurons were used for in vitro studies on days 12-14 (DIV 12-14) and verified to be greater than $95 \%$ viable.

\section{Antibodies and reagents}

A primary antibody against Preso was obtained from R\&D Systems, Inc. (Minneapolis, MN, USA). Antibodies against nNOS, NR1, NR2A, NR2B, and PSD-95 were obtained from NeuroMab (Davis, CA, USA). Antibodies against CDK5 and phospho-NR2B (Ser1284) were obtained from Cell Signaling Technology (Danvers, MA, USA). An antibody against $\beta$-actin was obtained from Sigma-Aldrich (St. Louis, MO, USA). For immunoblotting, HRP-conjugated anti-rabbit and anti-mouse secondary antibodies were used (Santa Cruz Biotechnology, CA, USA). The Alexa Fluor 488 mouse IgG and Alexa Fluor 594 rabbit IgG secondary antibodies (Thermo Fisher Scientific) were used for immunostaining. DL-AP5, MK-801, BAPTA-AM, ARL 17447, and purvalanol B were obtained from Tocris Bioscience (Bristol, UK). ZL006 was obtained from EMD Millipore (Billerica, MA, USA). TatNR2B9c was obtained from ProbeChem (St. Pete Beach, FL, USA).

\section{TBI models}

The in vitro model of TBI employed in the present study was previously described by Mukhin et al. with 
some modification ${ }^{29}$. This TNI employed a plastic stylet to scrape adherent cells from a culture dish, thereby tearing processes and soma while leaving a significant proportion of cells intact. This model was employed in the present study as described previously ${ }^{11}$. Briefly, each confluent cell culture was manually scratched with a sterile plastic pipette tip following a square grid (with $3 \mathrm{~mm}$ spacing between the lines). To reduce the inconsistency of damage in different experiments, all TNI models were established by the same researcher in our group using a standard square grid module. The cultured neurons were used for in vitro studies on DIV 12-14. Culture cells were placed in an incubator at $37^{\circ} \mathrm{C}$ until a designated posttrauma time point was reached, and the medium was not changed. Experiments were performed from $0 \mathrm{~h}$ (immediately after mechanical injury) to $24 \mathrm{~h}$ after trauma. Uninjured cell cultures were used as controls. Because scratch injury first activates neurons at the wound edge and later expands to the entire neuronal monolayer, the entire culture on each dish was used for all experiments.

A CCI model was used as the in vivo model of TBI in the present study. After induction of anesthesia with $4 \%$ isoflurane, a mouse was placed on a platform with a stereotactic frame. A midline longitudinal scalp incision was made, the skull was exposed, and the dura was kept intact. Injury was induced by impacting the right cortex with an actuator ( $3 \mathrm{~mm}$ diameter) at a rate of $3 \mathrm{~m} / \mathrm{s}$ and a compression of $1.5 \mathrm{~mm}$. Then, the scalp wound was closed with standard suture material, and the wound area was treated with lidocaine cream. The mice were returned to their cages at the end of the surgical procedures, where and water and food were available ad libitum.

\section{Preparation of lentivirus and adeno-associated virus for RNAi and overexpression}

All lentiviruses and adeno-associated viruses were developed and obtained from GeneChem Company (Shanghai, China). To develop the shRNA lentiviruses and shRNA adeno-associated virus (AAV), an siRNA oligo (CCTTGTGTCCCAAAGAGCA) was subcloned into a GV248 lentiviral vector (hU6-MCS-Ubiquitin-EGFPIRES-puromycin) (S1) and a GV478 AAV serotype 2 vector (U6-MCS-CAG-EGFP) (Fig. S2). To develop overexpression lentiviruses, cDNAs of Preso, Preso without the PSD-95-binding C-terminus (deletion of amino acids 1309-1312), CDK5, and Preso without the Ddomain (deletion of amino acids 641-655) were subcloned into a G492 lentiviral vector (Ubi-MCS-3FLAGCBh-gcGFP-IRES-puromycin) (Fig. S1). The cultured neurons were used for transfection on DIV 12-14. After transfection with different lentiviruses for $72 \mathrm{~h}$, neurons were prepared for further experiments. The AAV vector was injected into the cortex using a stereotaxic instrument. At $14 \mathrm{~d}$ after the cortical injection of AAV, mice were prepared for brain trauma.

\section{Western blot analysis}

After various treatments, mouse cortical neurons were washed with ice-cold phosphate-buffered saline (PBS) three times and lysed with buffer containing PhosSTOP protease inhibitor and phosphatase inhibitor mixture tablets (Roche Applied Bioscience, Indianapolis, IN, USA). The protein concentration in the supernatant was determined using a BCA protein kit. The proteins were separated on 10-15\% and 10\% SDS-PAGE gels and transferred to nitrocellulose membranes (Thermo Fisher Scientific). The membranes were soaked in a 5\% nonfat milk solution comprising Tris-buffered saline and $0.05 \%$ Tween 20 (TBST) for $1 \mathrm{~h}$ at room temperature and then incubated overnight at $4{ }^{\circ} \mathrm{C}$ with the appropriate primary antibodies (Preso, 1:500 dilution; NR1, 1:500 dilution; NR2A, 1:500 dilution; NR2B, 1:500 dilution; PSD-95, 1:1000 dilution; $\beta$-actin, 1:2500 dilution). The membranes were washed in TBST and incubated for $1 \mathrm{~h}$ at room temperature with the secondary antibodies diluted in blocking buffer. Immunoreactivity was detected by the SuperSignal West Pico Chemiluminescent Substrate (Thermo Fisher Scientific). The optical densities of the bands were quantified using an image analysis system with ImageJ (National Institutes of Health, MA, USA).

\section{LDH assay}

Cytotoxicity was determined by the release of LDH, a cytoplasmic enzyme released from cells and a marker of membrane integrity. LDH release into the culture medium was detected using an LDH cytotoxicity assay kit obtained from Cayman Chemical (Ann Abor, MI, USA) according to the manufacturer's instructions. Briefly, $100 \mu \mathrm{l}$ of supernatant from each well was collected in a new 96-well plate. LDH reaction solution was added to each well, and the mixture was incubated for $30 \mathrm{~min}$ at $37^{\circ} \mathrm{C}$ with gentle shaking. The activity of LDH was calculated from the absorbance measured at a wavelength of $490 \mathrm{~nm}$ with a plate reader.

\section{Cell death assessment}

Following the exposure of neuronal cultures to the various treatments, neuronal survival was quantified and presented as the percentage of cell death. Neuronal survival was determined by staining treated neuronal cultures with $5 \mu \mathrm{M}$ Hoechst 33342 and $2 \mu \mathrm{M}$ PI (Sigma-Aldrich) for $10 \mathrm{~min}$. Cell culture plates were placed on the mechanical stage of a Nikon microscope, and photomicrographs were acquired by a blinded observer. The numbers of total and injured (PI-positive) cells were counted by automated computer-assisted software 
(Image-Pro Plus version 6.0, Media Cybernetics, Inc.). The raw counts were analyzed in Microsoft Excel to determine the percent cytotoxicity and conduct statistical analysis.

\section{Measurement of intracellular nitric oxide}

Intracellular nitric oxide (NO) was estimated using 4amino-5-methylamino-2', $7^{\prime}$-difluorofluorescein (DAFFM) diacetate obtained from Molecular Probes (Eugene, OR, USA) according to the manufacturer's instructions. Briefly, neurons were prepared on slides, and a DMSO stock solution was diluted at a concentration of $10 \mu \mathrm{M}$. After the various treatments, neurons were incubated with DAF-FM diacetate for $60 \mathrm{~min}$ at room temperature. Then, the cells were washed to remove excess probe and incubated with fresh medium for $30 \mathrm{~min}$. Fluorescence data were acquired using a fluorescence plate reader at an excitation wavelength of $495 \mathrm{~nm}$ and an emission wavelength of $515 \mathrm{~nm}$.

\section{Calcium imaging}

The intracellular $\mathrm{Ca}^{2+}$ concentration was measured using the ratiometric calcium indicator Fura-2-AM (Thermo Fisher Scientific). Cultured neurons grown on glass slides were treated with $5 \mu \mathrm{M}$ Fura-2-AM for $45 \mathrm{~min}$ before various treatments at room temperature. The neurons were then placed in an open-bath imaging chamber containing Dulbecco's PBS $(0.901 \mathrm{mM} \mathrm{CaCl}$, $0.493 \mathrm{mM} \quad \mathrm{MgCl}_{2}-6 \mathrm{H}_{2} \mathrm{O}, \quad 2.67 \mathrm{mM} \quad \mathrm{KCl}, \quad 1.47 \mathrm{mM}$ $\mathrm{KH}_{2} \mathrm{PO}_{4}, 137.93 \mathrm{mM} \mathrm{NaCl}$, and $8.06 \mathrm{mM} \mathrm{Na}_{2} \mathrm{HPO}_{4}$ $7 \mathrm{H}_{2} \mathrm{O}, \mathrm{pH}$ 7.2-7.4) supplemented with $20 \mathrm{mM}$ glucose at ambient temperature. Using a Nikon inverted epifluorescence microscope, the neurons were excited at wavelengths of 345 and $385 \mathrm{~nm}$, and fluorescence images were acquired at an emission wavelength of $510 \mathrm{~nm}$. The images were acquired and analyzed with MetaFluor image processing software. The $\mathrm{Ca}^{2+}$ concentration values were then calculated, and nonspecific $\mathrm{Ca}^{2+}$ fluorescence was subtracted from each wavelength before the calculations were conducted.

\section{Coimmunoprecipitation (Co-IP)}

To evaluate protein interactions, Co-IP experiments were performed using a Pierce ${ }^{\mathrm{mu}}$ Crosslink Magnetic IP/ Co-IP Kit (Thermo Fisher Scientific) according to the manufacturer's instructions. Cortical neurons were cultured in 100-mm dishes and then harvested in ice-cold lysis/wash buffer supplemented with a proteinase inhibitor cocktail (Roche Applied Bioscience). The lysate was centrifuged at $13,000 \times g$ for $15 \mathrm{~min}$ at $4{ }^{\circ} \mathrm{C}$. Protein concentrations in the extracts were determined using a BCA protein assay kit (Thermo Fisher Scientific). Magnetic beads were crosslinked with nonspecific mouse IgG (2 mg), anti-PSD-95 (NeuroMab) and anti-CDK5 (Cell Signaling Technology), and the beads were then washed two times with coupling buffer. The protein extracts were combined with the beads and incubated overnight at $4{ }^{\circ} \mathrm{C}$. Following magnetic isolation, the precipitates were washed three times with wash buffer, eluted with elution buffer, neutralized with neutralization buffer, and prepared for western blot and kinase activity assays.

\section{CDK5 activity assay}

After various treatments, immunoprecipitants were obtained by CDK 5 immunoprecipitation. A CDK 5 activity assay was performed using the ADP-Glo ${ }^{\text {tw }}$ Kinase Assay Kit (Promega Corporation, WI, USA) according to the manufacturer's protocol. Briefly, enzyme, substrate, ATP and inhibitors were diluted in Kinase Buffer. The inhibitor, enzyme, and substrate/ATP mixture were added to the 96-well plate and incubated at room temperature for $10 \mathrm{~min}$. Then, ADP-Glo ${ }^{\mathrm{mi}}$ Reagent was added to each well of the plate, and the mixture was incubated at room temperature for $40 \mathrm{~min}$. Next, Kinase Detection Reagent was added to each well of the plate, and the mixture was incubated at room temperature for $30 \mathrm{~min}$. The results were acquired by measuring the luminescence with a plate-reading luminometer.

\section{Stereotaxic injection of lentivirus}

The in vivo delivery of AAV-shPreso and Vector was carried out using stereotaxic cortical injection as previously described ${ }^{11}$. Three cortical injections were performed in the right hemisphere (ipsilateral to the lesion) as follows: point $1,1.0 \mathrm{~mm}$ anterior to the bregma, $1.5 \mathrm{~mm}$ lateral, and $1.5 \mathrm{~mm}$ deep; point 2, $0 \mathrm{~mm}$ antero-posterior to the bregma, $1.5 \mathrm{~mm}$ lateral, and $1.5 \mathrm{~mm}$ deep; and point $3,1.0 \mathrm{~mm}$ posterior to the bregma, $1.5 \mathrm{~mm}$ lateral, and $1.5 \mathrm{~mm}$ deep. All the target points were in the right hemisphere (ipsilateral to the lesion). Each injection contained $1.5 \mu \mathrm{l}$ of $1 \times 10^{9} \mathrm{TU} / \mathrm{ml}$ AAV suspension, which was injected at a rate of $0.2 \mu \mathrm{l} / \mathrm{min}$. The needle was withdrawn over the course of $10 \mathrm{~min}$. Fourteen days after the lentivirus injection, mice were subjected to brain trauma as described above.

\section{Neurological severity score (NSS)}

The NSS, which is highly correlated with the severity of brain damage, was measured as previously described ${ }^{30}$. Neurological severity scoring was conducted by an investigator who was blinded to the experimental groups. The score consists of 10 individual neurological parameters, including tasks on motor function, alertness and physiological behavior. One point was awarded for the 
lack of a tested reflex or for the inability to perform a task, and no points were awarded for success. A maximal NSS of 10 points indicated severe neurological dysfunction, with failure of all tasks.

\section{Water content measurement}

The extent of cerebral edema was evaluated by determining the tissue water content in the injured hemisphere as previously reported ${ }^{31}$. Briefly, mice were decapitated under deep anesthesia with $100 \mathrm{mg} / \mathrm{kg}$ pentobarbital. Their brains were quickly removed, and the hemispheres were separated in the sagittal plane. Coronal sections with a thickness of $3 \mathrm{~mm}$ were prepared from the area bordering the lesion. These sections were weighed (wet weight) and dried in a desiccator oven for $24 \mathrm{~h}$ at $95^{\circ} \mathrm{C}$. After weighing the dried sections (dry weight), the water contents of the cortices ipsilateral to the injury (Wi) and contralateral to the injury (Wc) were calculated using equation one as follows: Water content (g/g dry weight) $=($ wet weight - dry weight $) /$ dry weight. Next, the difference between Wi and Wc was calculated via equation two as follows: \% Change in water content $=[(\mathrm{Wi}-\mathrm{Wc}) \times$ 100]/Wc.

\section{Statistical analysis}

All experiments were performed a minimum of three times. Statistical evaluation was performed with GraphPad Prism software, version 6.0 (GraphPad, San Diego, CA). Significant differences between experiments were assessed by univariate ANOVA (more than two groups) followed by Bonferroni's multiple comparison or unpaired t tests (two groups).

\section{Acknowledgements}

This work was supported by the National Natural Science Foundation of China (nos. 81771322, 81601149, 81601077, 81771239, 81430043), the Military Youth talent lifting project (no. 17-JCJQ-QT-037), the Youth talent lifting project of Shaanxi province (20180305), the Natural Science Foundation of Shaanxi province (2017JQ8038), and a project funded by the China Postdoctoral Science Foundation (2015M572683).

\section{Author details}

${ }^{1}$ Department of Neurosurgery, Xijing Hospital, Fourth Military Medical University, Xi'an, China. ${ }^{2}$ Department of Biomedical Engineering, Fourth Military Medical University, Xi'an, China. ${ }^{3}$ Department of Anesthesiology, Xijing Hospital, Fourth Military Medical University, Xi'an, China

\section{Conflict of interest}

The authors declare that they have no conflict of interest.

\section{Publisher's note}

Springer Nature remains neutral with regard to jurisdictional claims in published maps and institutional affiliations.

Supplementary Information accompanies this paper at (https://doi.org/ 10.1038/s41419-019-1731-x).
Received: 27 January 2019 Revised: 25 May 2019 Accepted: 4 June 2019 Published online: 24 June 2019

References

1. Maas, A. I. R. et al. Traumatic brain injury: integrated approaches to improve prevention, clinical care, and research. Lancet Neurol 16, 987-1048 (2017).

2. Loane, D. J. \& Faden, A. I. Neuroprotection for traumatic brain injury: translational challenges and emerging therapeutic strategies. Trends Pharmacol Sci 31, 596-604 (2010).

3. Luo, P., Fei, F., Zhang, L., Qu, Y. \& Fei, Z. The role of glutamate receptors in traumatic brain injury: implications for postsynaptic density in pathophysiology. Brain Res Bull 85, 313-320 (2011).

4. Sommer, J. B. et al. In vitro and in vivo effects of a novel dimeric inhibitor of PSD-95 on excitotoxicity and functional recovery after experimental traumatic brain injury. Eur J Neurosci 45, 238-248 (2017).

5. Lee, H. W. et al. Preso, a novel PSD-95-interacting FERM and PDZ domain protein that regulates dendritic spine morphogenesis. The. J Neurosci 28, 14546-14556 (2008).

6. $\mathrm{Hu}, \mathrm{J}$. H. et al. Preso 1 dynamically regulates group I metabotropic glutamate receptors. Nat Neurosci 15, 836-844 (2012).

7. Luo, P. et al. Downregulation of postsynaptic density-95-interacting regulator of spine morphogenesis reduces glutamate-induced excitotoxicity by differentially regulating glutamate receptors in rat cortical neurons. FEBS $J \mathbf{2 8 0}$, 6114-6127 (2013)

8. Mo, J. et al. Preso regulation of dendritic outgrowth through PI(4,5)P2dependent PDZ interaction with betaPix. Eur J Neurosci 36, 1960-1970 (2012).

9. Hu, J. H., Worley, P. F. \& Kammermeier, P. J. Dynamic regulation of homer binding to group i metabotropic glutamate receptors by Presol and converging kinase cascades. J Pharmacol Exp Ther 361, 122-129 (2017).

10. Sommer, J. B. et al. Effects of dimeric PSD-95 inhibition on excitotoxic cell death and outcome after controlled cortical impact in rats. Neurochem Res $\mathbf{4 2}$, 3401-3413 (2017)

11. Luo, P. et al. Postsynaptic scaffold protein Homer 1a protects against traumatic brain injury via regulating group I metabotropic glutamate receptors. Cell Death Dis 5, e1174 (2014).

12. Shohami, E. \& Biegon, A. Novel approach to the role of NMDA receptors in traumatic brain injury. CNS Neurol Disord Drug Targets 13, 567-573 (2014).

13. Khaldi, A., Chiueh, C. C., Bullock, M. R. \& Woodward, J. J. The significance of nitric oxide production in the brain after injury. Ann N Y Acad Sci 962, 53-59 (2002).

14. Besson, V. C. Drug targets for traumatic brain injury from poly(ADP-ribose) polymerase pathway modulation. Br J Pharmacol 157, 695-704 (2009).

15. Cook, D. J., Teves, L. \& Tymianski, M. Treatment of stroke with a PSD-95 inhibitor in the gyrencephalic primate brain. Nature 483, 213-217 (2012).

16. Zhou, L. et al. Treatment of cerebral ischemia by disrupting ischemia-induced interaction of nNOS with PSD-95. Nat Med 16, 1439-1443 (2010).

17. Chen, $X$. et al. PSD-95 is required to sustain the molecular organization of the postsynaptic density. J Neurosci 31, 6329-6338 (2011).

18. Kucharz, K., Sondergaard Rasmussen, I., Bach, A., Stromgaard, K. \& Lauritzen, M. PSD-95 uncoupling from NMDA receptors by Tat- N-dimer ameliorates neuronal depolarization in cortical spreading depression. J Cereb Blood Flow Metab 37, 1820-1828 (2017).

19. Bach, A. et al. Biochemical investigations of the mechanism of action of small molecules ZL006 and IC87201 as potential inhibitors of the nNOS-PDZ/PSD95-PDZ interactions. Sci Rep 5, 12157 (2015).

20. Luo, C. X. et al. Interaction of nNOS with PSD-95 negatively controls regenerative repair after stroke. The. J Neurosci 34, 13535-13548 (2014).

21. Shah, K. \& Lahiri, D. K. Cdk5 activity in the brain-multiple paths of regulation. J Cell Sci 127(Pt 11), 2391-2400 (2014).

22. Yousuf, M. A. et al. Involvement of aberrant cyclin-dependent kinase 5/p25 activity in experimental traumatic brain injury. J Neurochem 138, 317-327 (2016).

23. Murphy, J. A. et al. Phosphorylation of Ser1166 on GluN2B by PKA is critical to synaptic NMDA receptor function and $\mathrm{Ca} 2+$ signaling in spines. The. J Neurosci 34, 869-879 (2014).

24. Chen, B. S. \& Roche, K. W. Regulation of NMDA receptors by phosphorylation. Neuropharmacology 53, 362-368 (2007) 
25. Li, B. S. et al. Regulation of NMDA receptors by cyclin-dependent kinase-5. Proc Natl Acad Sci USA 98, 12742-12747 (2001).

26. Plattner, F. et al. Memory enhancement by targeting $C \mathrm{dk} 5$ regulation of NR2B. Neuron 81, 1070-1083 (2014).

27. Lu, W. et al. A novel phosphorylation site of $\mathrm{N}$-methyl-D-aspartate receptor GluN2B at S1284 is regulated by Cdk5 in neuronal ischemia. Exp Neurol $\mathbf{2 7 1}$ 251-258 (2015).

28. $\mathrm{Ai}_{1} \mathrm{H}$. et al. Acute stress regulates phosphorylation of $\mathrm{N}$-methyl-d-aspartate receptor GluN2B at S1284 in hippocampus. Neuroscience 351, 24-35 (2017).
29. Chen, T. et al. Down-regulation of Homer1b/c attenuates glutamate-mediated excitotoxicity through endoplasmic reticulum and mitochondria pathways in rat cortical neurons. Free Radic Biol Med 52, 208-217 (2012).

30. Flierl, M. A. et al. Mouse closed head injury model induced by a weight-drop device. Nat Protoc 4, 1328-1337 (2009).

31. Keep, R. F., Hua, Y. \& Xi, G. Brain water content. A misunderstood measurement? Transl Stroke Res 3, 263-265 (2012). 